\title{
Ectodermal Dysplasia
}

\author{
Vassa Khema Katthika ${ }^{1}$, Elza Ibrahim Auerkari ${ }^{2}$ \\ ${ }^{1}$ Pediatric Dentistry Residency Program, Faculty of Dentistry, Universitas Indonesia, Jakarta, \\ Indonesia \\ ${ }^{2}$ Department of Oral Biology, Faculty of Dentistry, Universitas Indonesia, Jakarta, Indonesia \\ *Email: elza.ibrahim@ui.ac.id
}

\begin{abstract}
Primary defects in the development of two or more ectoderm-derived tissues are shared by a rare heterogeneous group of inherited disorders called ectodermal dysplasia (ED) syndrome. The affected tissues are primarily the hair, skin, teeth, sweat glands, and nails. The most common phenotype of ED is hypohidrotic ED (HED), which is characterized by hypohidrosis/anhidrosis, hypotrichosis, and hypodontia/anodontia. Other clinical features of HED include frontal bossing with prominent supraorbital ridges, nasal bridge depression, and protuberant lips. There are three subtypes of HED: autosomal recessive, autosomal dominant, and Xlinked. Mutations to four genes (EDA, EDAR, EDARADD, and WNT10A) are responsible for most cases of ED. Besides gene mutations, epigenetics also influences gene expression and activity in ED syndrome. DNA methylation and histone modification are vital to the epigenetic regulation of the development of female carriers of the X-linked subtype of HED.
\end{abstract}

Keywords: ectodermal dysplasia, mutation, EDA gene, EDAR gene, EDARADD gene

\section{Introduction}

At about day 21 of development, the gastrulation process results in the formation of three primitive embryonic components, the ectoderm, mesoderm, and endoderm. Of these, the ectoderm undergoes subdivision into the neuroectoderm, which gives rise to the early nervous system. At this time, the entire embryonic surface is enveloped by the ectoderm, which also gives rise to the tooth enamel, epidermal appendages, and epidermis [1]. The development of such multipurpose and complex structures is regulated by an extremely coordinated interaction of numerous genetic signaling pathways within and between the mesenchymal and ectodermal layers of the embryonic tissue. Malfunction at any point during the development of these ectodermal structures will result in phenotypical variations of specific entities, in a syndrome known as ectodermal dysplasia (ED) [2].

ED describes a range of inheritable disorders that are characterized by particular shared functional and structural anomalies in ectoderm-derived tissues. Any of the possible Mendelian inheritance patterns can transmit these rare conditions. ED can also affect the retina and lens, as well as the skin, parts of the inner ear, the nerves, development of the fingers and toes, and other parts of the body based on the particular syndrome. Individual syndromes are characterized by distinct combinations of symptoms that usually manifest from mild to severe. At present, combination of 
about 30 causative gene mutations have been associated with more than 200 different ED syndromes [1].

\section{Definition and History}

ED encompasses a large group of hereditary disorders caused by primary defects in the development of two or more tissues derived from the ectoderm. The affected tissues are the hair, skin, teeth, sweat glands, and nails [3]. Danz first recorded ED in 1792 . In 1838, ED was documented in 10 Hindu male family members by Wedderburn. Moreover, two cases of the hypohidrotic form of ED were reported by Thurnam in 1848, and Guilford and Hutchinson reported similar cases in 1883 and 1886, respectively. The term "hereditary ED” was introduced by Weech in 1929, who also proposed the term "anhidrotic" to describe those with the incapability to perspire. Because persons with the hypohidrotic form of ED are not actually devoid of eccrine glands, Felsher proposed changing the term "anhidrotic" to "hypohidrotic" in 1994 [2].

\section{Classification}

ED describes a diffuse range of nonprogressive, congenital disorders with more than 200 distinctive syndromes identified to date. A specific ED-related syndrome is classified on the basis of unique clinical features composed of a combination of ectodermal defects and other anomalies, while "pure ED" is characterized by defects only to the ectodermal structures [4].

The most common phenotypes of ED are hidrotic, also known as Clouston syndrome, and hypohidrotic (anhidrotic), which is also known as Christ-Siemens-Touraine syndrome [5]. The most common phenotype is hypohidrotic ED (HED), which is characterized by abnormal development of the teeth (anodontia or hypo dontia), hair (hypotrichosis), and sweat glands (anhidrosis or hypohidrosis) [2,6].

The dental manifestations of HED include abnormal crown formation (conical shape or pegged teeth), delayed eruption of the permanent teeth, hypoplastic labial frenum, and a wide midline diastema in the autosomal recessive form. Other clinical features of HED include frontal bossing, prominent supraorbital ridges, depression of the nasal bridge and midface, prominent and obliquely set ears, protuberant lips, and a lack of alveolar bone development causing the lower third of the face to appear small [5]. There are three types of HED: (1) autosomal recessive, (2) autosomal dominant, and (3) X-linked HED (XLHED) [7].

\section{$4 \quad$ Etiology and Prevalence}

HED is caused by mutations in any of the three ectodysplasin pathway genes: (1) ectodysplasin A $(E D A)$, which encodes a ligand for the (2) EDA receptor $(E D A R)$, and (3) the EDAR-associated death domain (EDARADD), which encodes an intracellular signaling mediator of this single linear pathway [5]. A new gene, WNT10A, was recently identified and suggested as one of the genes responsible for the various autosomal recessive forms of ED [8]. In rare cases, mutations in further downstream 
components of the EDA pathway cause a form of HED that is associated with immune deficiency [5].

The prevalence of HED is estimated at one case for every 17,000 persons worldwide [5]. According to McKusick, any form of ED is rare with an incidence of approximately seven cases per 10,000 births [9]. with a prevalence ranging from one in 10,000 to 100,000 live male births $[2,4]$.

\section{Genetic Inheritance Patterns}

Mutations or deletions of certain genes located on other chromosomes have also been associated with the familial inheritance of several types of ED. However, in some cases, ED has been known to affect individuals with no familial history of the disease, as with a case of a de novo mutation reported by Deshmukh and Prashanth [2]. Most cases of HED show an X-linked recessive inheritance pattern, although cases of autosomal recessive and dominant inheritance patterns have also been reported [10].

\section{HED-Associated Genes and Its Function}

Most cases of HED are associated with mutations to genes involved in the ectodysplasin pathway (i.e., EDA, EDAR, EDARADD, and $W N T 10 A$ ) [8]. The EDA protein, which is encoded by the $E D A$ gene [11] is a type 2 membrane protein of the tumor necrosis factor (TNF) family composed of 391 amino acids. Ectodysplasin A1 (EDA-A1), which binds to EDAR, and ectodysplasin A2 (EDA-A2), which binds to the ligand of X-linked EDAR A2, are the most important isoforms of EDA.

The EDAR gene encodes EDAR, which is a type 1 transmembrane protein belonging to the TNF receptor superfamily that is composed of 448 amino acids. EDAR has a transmembrane region, a death domain (DD) in its intracellular region, and an extracellular region [12].

A DD is a protein interaction module that interacts with the DDs of other proteins and generates metabolic cascades that are frequently involved in the regulation of inflammation and apoptosis through the NF-кB cascade. EDA-A1 binds to the extracellular domain of EDAR, while apoptotic transduction signals are initiated by the association of the DD with the intracellular region of EDARADD $[1,10]$.

The EDARADD gene encodes the 208-amino acid sequence, the EDARassociated DD protein [13]. The normal interaction between these two proteins and the subsequent regulation of embryonic morphogenesis are dependent on the integration of the DDs. Thus, it is important that the $E D A$-mediated signaling pathway is appropriate for the development of organs and ectoderm-derived structures, such as the mammary glands, nails, hair, pituitary gland, eyes, nose, tooth enamel, and sweat glands [1].

\section{Genetic Pathogenesis}

Defective ectodysplasin, which results from mutations to the $E D A, E D A R$, or $E D A R A D D$ gene, prevents normal interactions between the mesoderm and ectoderm and results impairment to the normal development of the teeth, hair, and sweat 
glands. The characteristic features of HED are induced by improper formation of these ectodermal structures [2].

\section{EDA Gene Mutation}

Mutations to the EDA gene (also known as EDl) are the most common cause of XLHED [14] with an incidence of approximately 1:100,000 births, which accounts for approximately $95 \%$ of HED cases $[1,2]$. The locus of the mutated $E D A$ gene is on the long arm of the X chromosome at Xq12-q13 $[1,5,15]$.

In regard to the heritance pattern of HED, the EDA gene is carried by females and manifested in males. The X-linked inheritance pattern occurs when the mutated gene is located on one of the two $\mathrm{X}$ chromosomes of the female. If both copies of the gene are mutated, the disorder will present in females. On the contrary, if the mutated gene is carried by only one $\mathrm{X}$ chromosome in each cell, the disorder will present in males, as males have only one $\mathrm{X}$ chromosome.

In other words, the daughter of a male with the XLHED genotype will be a carrier because of the transmission of the $E D A$ allele from her father. She may also exhibit a mild manifestation of ED. However, his son will never be affected. In contrast, half of the offspring of woman with the XLHED genotype will be affected regardless of gender. Therefore, $50 \%$ of her daughters will be carriers and $50 \%$ of her sons will be affected. The carrier daughters may exhibit mild manifestations, such as pegged-shaped teeth, hypodontia, and reduced sweat excretion [2].

During the induction process of epithelial-mesenchymal transition, the mutant $E D A$ affects cell migration or cell signaling transduction [14]. Exons 3 and 5-9 are the most frequent locations of the mutation to the $E D A$ gene [16]. The occurrence of an insertion or deletion of genetic material and alteration in single DNA base pairs are the result of mutations to the $E D A$ gene, which causes the formation of a nonfunctional protein. Non-stimulating chemical signals result from this abnormal protein formation, as normal signaling is required for the interaction between the mesoderm and ectoderm structures. So without these signals, the teeth, sweat glands, hair follicles, and other ectodermal structures are not formed properly, which manifests into the clinical features of HED [15].

Only one $E D A$ mutation accounts for $80 \%$ of $H E D$ cases, even though many studies have reported a wide range of insertions and deletions. Moreover, more than 204 distinctive mutations of the $E D A$ gene have been identified [1], with several having been detected in Chinese populations. In a recent study, Zeng et al. identified eight mutations in seven unrelated HED families. Of these eight mutations, two were novel: a heterozygous mutation of the $E D A$ gene found in families 1-5, and one mutation of the $E D A R$ gene identified in family 6 . In addition, there were two mutations of WNT1OA and two single-nucleotide polymorphisms of EDAR in family 7.

The collagen-like domain of the EDA protein is the site of a novel mutation of $E D A$ (i.e., c.662G $>$ A (p.Gly221Asp). The collagen-like domain functions as a connector for EDA trimerization, which has been interfered by point mutations, of which about $86 \%$ (12 of 14) are glycine mutations, indicating the necessity of glycine in maintaining the function and structure of the collagen-like domain. The mutation c. $662 \mathrm{G}>\mathrm{A}$ causes a replacement of a glycine residue with aspartic acid in 
the collagen-like domain, which results in a mutated protein that may not achieve trimerization.

A cohort study about the distribution of $E D A$ mutations established that each of these mutations occurred in the collagen-like domain, furin sites, of the TNFbinding domain, except c.741G $>$ A (p.Gln247Gln). Because the c. $741 \mathrm{G}>\mathrm{A}$ mutation is located prior to the TNF domain, the functional domain may also be affected, which results in alterations to the splicing of the EDA transcript [7].

\section{EDAR Gene Mutation}

Mutations to the EDAR gene occur precisely at locus 2q11-q113 of the long arm of chromosome 2. The EDAR gene has 12 exons and encodes the EDAR protein [12]. So far, of the 41 mutations to $E D A R$ that have been identified to date, most involve exon 12. The mutated gene encodes the $\mathrm{C}$-terminal region of the receptor protein (the location of the DD) [1] which contains a single amino acid substitution. In addition, deletions of genetic material from the $E D A R$ gene also occur. The production of an abnormal receptor of EDA is induced by several $E D A R$ mutations. As a result of these genetic changes, the signaling pathway necessary for the interactions of the mesoderm-ectoderm structures and normal formation of the ectodermal structures, such as sweat glands and hair follicles, is disrupted [17].

$E D A R$ gene mutations are responsible for the autosomal dominant and autosomal recessive forms of HED. In the autosomal dominant form of HED, one copy of the mutated $E D A R$ gene is present in each cell, and this single alteration is sufficient to cause problems in a carrier. In contrast, in the autosomal recessive form of HED, two copies of the mutated $E D A R$ gene are present in each cell. It is frequently found that the asymptomatic parents of a person with an autosomal recessive disorder are carriers who each bring one copy of the mutated gene $[17,18]$.

Shimomura et al [10] found two novel heterozygous EDAR mutations in a Japanese female patient affected with HED, including one that produces an amino acid substitution in the DD of the EDAR: a heterozygous $\mathrm{G} \rightarrow \mathrm{A}$ transition occurring at the first base pair of intron 2 of the EDAR gene (exon 2 IVS $+1 \mathrm{G} \rightarrow \mathrm{A}$ ). The splice-donor-site mutation at the $5^{\prime}$-end of intron 2 (Fig. 8) was found only in the genomic DNA of the patient, but not her parents. A heterozygous $\mathrm{G} \rightarrow \mathrm{A}$ transition $(1124 \mathrm{G} \rightarrow \mathrm{A})$, which occurs in exon 12 of the EDAR gene, was the second novel mutation, which results a $\mathrm{R} 375 \mathrm{H}$ substitution at the protein level that is predicted to generate a substitution of arginine by histidine at amino acid residue 375 in exon 12 . This mutation was also found to have been inherited from the patient's unaffected father.

Initially, Chassaing et al. found that about $25 \%$ of EDAR mutations are nonED1-related. The recessive effect will be obtained if the mutations lead to a premature stop codon, unless the stop codon is in the last exon, whereas the dominant negative effect, which results in many variations in expression, will be obtained if a heterozygous missense mutation occurs in the functional domain. A more severe phenotype occurs in patients with compound heterozygous or homozygous EDAR mutations than in those with heterozygous nonsense, frameshift, or missense mutations [19]. 


\section{EDARADD Gene Mutation}

The EDARADD gene, which encodes the EDARADD protein, is located on chromosome 1 at locus 1q42-q43 [1,19]. Acting as an adapter in triggering chemical signals within celsl, the EDARADD protein interacts with the EDAR [18]. The mutation of EDARADD gene, which occurs in the DD, causes a single amino acid substitution of a glutamine residue with a lysine residue at position 142 (Glu142Lys) of the EDARADD protein. This mutation prevents normal interactions between the EDAR and EDARADD protein. In this scenario, the receptor is unable to trigger signals that are required for mesoderm-ectoderm interactions as well as the normal development of sweat glands, hair follicles, and other ectodermal structures [13] This mutation of the EDARADD gene is related with the autosomal recessive pattern of HED inheritance. In fact, this autosomal recessive form of HED has been identified in a family with a homozygous missense mutation of the EDARADD gene [10].

\section{WNT10A Gene Mutation}

The WNT10A gene, which is located at locus 2 q35 on chromosome 2, encodes a large family of glycoproteins involved in the Wnt signaling pathway that play important roles in the development and differentiation of cells derived from ectodermal tissue during the adult and embryogenic stages [1,7]. Mutations of the WNT10A gene have been suggested to generate some forms of autosomal ED inheritance. HED, Schöpf-Schulz-Passarge syndrome (SSPS), and odonto-onycho-dermal dysplasia (OODD) are examples of the autosomal forms of ED. One of the rarest forms of ED is OODD. Its characteristics are especially found in palmoplantar keratoderma, the formation of abnormal teeth, hypotrichosis, nail dystrophy, and many other symptoms, including neoplasms related to ectodermal abnormalities. SSPPS is another form of ED similar to OODD, which can be differentiated by the presence of cysts in the eyelid [20].

According to a recent study by Zeng [7], SSPS and OODD are the same disease with varying symptoms caused by mutations of the WNT10A gene. HED has milder manifestations than OODD, but more severe than oligodontia. They consider that HED, oligodontia, and OODD describe the whole clinical manifestations of ED in relation to $W N T 10 A$ mutations. It is rare that the same mutated gene causes different phenotypes. In this study, Zeng found two mutations of the $W N T 10 A$ gene: a novel c.354T $>\mathrm{G}$ (p.Tyr118*) mutation and the previously described c.637G $>$ A (p.Gly213Ser) mutation.

\section{Epigenetics}

Epigenetics is the study of meiotic and mitotic changes in the control of gene expression that can be inherited without changes to nucleotide sequences in the genome [21]. Epigenetics sometimes indicate changes in a chromosome that influence gene expression and activity. The effects on physiological and cellular phenotypic traits may be part of the normal developmental program (flexible and dynamic response to extracellular or intracellular stimuli) or can be affected by environmental or external factors. For example, alterations in hormonal levels, cytokines, response 
of stress, growth factors, and neurotropic factors are modulated by environmental factors [22]. Epigenetics can be basically defined as inheritable changes in the cells of offspring. The term epigenetics also refers to the changes themselves, that is, functional genomic changes that do not involve changes in the nucleotide sequence, such as DNA methylation and histone modification. Each of these modifications changes how genes are expressed without changing the main sequence of the DNA [23].

DNA methylation is the process of adding a methyl group to a cytosine residue occurring just before a guanine residue by DNA methyltransferases. This process is biological and occurs after DNA duplication. It has been reported that in living cells, methylation is the most common type of DNA modification and has both mutagenic and epigenetic effects on chromatin inactivation, gene expression, cell differentiation, oncogenesis, and embryonic growth.

$E D A$ contains a large $\mathrm{CpG}$ island in its promoter, which acts as the main target of DNA hypermethylation, and specific or regional methylation events interfere with transcription. Hypermethylation of the $\mathrm{CpG}$ island promoter assists in gene silencing by remodeling the chromatin, preventing the bonding of specific transcription factors to their recognition sequence, and attracting methylated DNA-binding proteins [14].

Histone modification is the post-translational change to the histone tail involving flexible stretches of $\mathrm{C}$ or $\mathrm{N}$ terminal residues extending from the globular histone octamers [22]. It is becoming more and more obvious in the post-genomic era that epigenetic control of gene expression plays an important role in determining the phenotype. Histone alterations and DNA methylation-demethylation events are vital to epigenetic regulation during the development of female carriers of the $\mathrm{X}$ linked form of HED. These alterations also result in mosaics of functionally normal and abnormal cells. However, whether $E D A$ promoter methylation contributes at all to the phenotype of XLHED carriers remains unclear. With the use of a quantitative pyrosequencing method to analyze the methylation level of the $E D A$ promoter, the Chinese carriers of XLHED have a hypermethylated $E D A$ promoter [24]; some with a methylation level $50 \%$ higher than in normal controls. There are no regular patterns among the mutation sites, methylation states, clinical features, or mutation types, although hypermethylated carriers are more inclined to have conical-shaped teeth and nail dysplasia than the hypomethylated group [14].

\section{Conclusion}

ED is a large hereditary group of disorders caused by primary defects in the development of two or more tissues derived from the ectoderm. The affected tissues are the hair, skin, teeth, sweat glands, and nails. HED is the most common phenotype of ED, which is a congenital syndrome characterized by hypohidrosis, hypotrichosis, and hypodontia. HED is mainly caused by mutations of four genes: EDA on chromosome Xq12-q13.1, which accounts for the X-linked form, EDAR on chromosome 2q11-q13 and EDARADD on chromosome 1q42-q43, which cause both the autosomal recessive and dominant forms, and $W N T 10 A$ on chromosome $2 \mathrm{q} 35$, which results in the autosomal recessive form. Besides these genetic factors, epige- 
netic factors, such as histone modification and DNA methylation, control gene expression. These modifications play significant roles in the establishment of the HED phenotype, while hypermethylation of the EDA promoter occurs in the X-linked form of HED.

\section{References}

1. García-Martín P, Hernández-Martín A, Torrelo A. Ectodermal dysplasias: a clinical and molecular review. Actas Dermo-Sifiliográficas (English Edition). 2013;104(6):451-70.

2. Deshmukh S, Prashanth S. Ectodermal dysplasia: a genetic review. Int J Clin Ped Dent. $2012 ; 5(3): 197$.

3. Weech AA. Hereditary ectodermal dysplasia (congenital ectodermal defect): a report of two cases. Am J Dis Child. 1929;37(4):766-90.

4. Mehta G. Ectodermal Dysplasia: Case report \& literature review. Eur J Dent Ther Res. 2013;3(1):170-173.

5. Kumar K, Shetty DC, Dua M, Dua A, Dhanapal R. An insight into the genesis of hypohidrotic ectodermal dysplasia in a case report. Case reports in dentistry. 2012;2012.

6. Zeng B, Lu H, Xiao X, Zhou L, Lu J, Zhu L, Yu D, Zhao W. Novel EDA mutation in $\mathrm{X}$ - linked hypohidrotic ectodermal dysplasia and genotype - phenotype correlation. Oral Dis. 2015;21(8):994-1000.

7. Zeng B, Xiao X, Li S, Lu H, Lu J, Zhu L, Yu D, Zhao W. Eight mutations of three genes (EDA, EDAR, and WNT10A) identified in seven hypohidrotic ectodermal dysplasia patients. Genes. 2016;7(9):65.

8. Cluzeau C, Hadj - Rabia S, Jambou M, Mansour S, Guigue P, Masmoudi S, Bal E, Chassaing N, Vincent MC, Viot G, Clauss F. Only four genes (EDA1, EDAR, EDARADD, and WNT10A) account for $90 \%$ of hypohidrotic/anhidrotic ectodermal dysplasia cases. Hum Mutation. 2011 Jan 1;32(1):70-2.

9. McKusick VA. Mendelian inheritance in man: a catalog of human genes and genetic disorders. JHU Press; 1998 Jun 29.

10. Shimomura Y, Sato N, Miyashita A, Hashimoto T, Ito M, Kuwano R. A rare case of hypohidrotic ectodermal dysplasia caused by compound heterozygous mutations in the EDAR gene. J Invest Dermatol. 2004;123(4):649-55.

11. National Library of Medicine. Genetic Home Reference [Internet] U.S. https://ghr.nlm.nih.gov/gene/EDA.

12. Monreal AW, Ferguson BM, Headon DJ, Street SL, Overbeek PA, Zonana J. Mutations in the human homologue of mouse dl cause autosomal recessive and dominant hypohidrotic ectodermal dysplasia. Nature Gen. 1999 Aug;22(4):366.

13. National Library of Medicine. Genetic Home Reference [Internet]. U.S. Available from: https://ghr.nlm.nih.gov/gene/EDARADD.

14. Yin W, Ye X, Fan H, Bian Z. Methylation state of the EDA gene promoter in Chinese X-linked hypohidrotic ectodermal dysplasia carriers. PloS one. 2013;8(4): e62203.

15. EDA gene [Internet]. Available from: https://ghr.nlm.nih.gov/gene/EDA.

16. Ohashi M, Moriya C, Tanahashi K, Nakano H, Sawamura D, Seishima M. A new EDA gene mutation in a family of X-linked hypohidrotic ectodermal dysplasia. J Dermatol Sci. 2014 1;74(2):175-7.

17. National Library of Medicine. Genetic Home Reference [Internet]. U.S. Available from: https://ghr.nlm.nih.gov/gene/EDAR.

18. National Library of Medicine. Genetic Home Reference [Internet]. Hypohidrotic ectodermal dysplasia [Internet]. Genetics Home Reference. 2018. Available from: https://ghr.nlm.nih.gov/condition/hypohidrotic-ectodermal-dysplasia\#inheritance.

19. Van Der Hout AH, Oudesluijs GG, Venema A, Verheij JB, Mol BG, Rump P, Brunner 
HG, Vos YJ, Van Essen AJ. Mutation screening of the Ectodysplasin-A receptor gene EDAR in hypohidrotic ectodermal dysplasia. Eur J Hum Gen. 2008;16(6):673.

20. He H, Han D, Feng H, Qu H, Song S, Bai B, Zhang Z. Involvement of and interaction between WNT10A and EDA mutations in tooth agenesis cases in the Chinese population. PloS one. 2013;8(11):e80393.

21. Osorio JC, Castillo A. Epigenetic Mechanisms in Head and Neck Cancer. InNew Aspects in Molecular and Cellular Mechanisms of Human Carcinogenesis 2016. InTech.

22. Kanherkar RR, Bhatia-Dey N, Csoka AB. Epigenetics across the human lifespan. Frontiers Cell Develop Bio. 2014;2:49.

23. Berger SL, Kouzarides T, Shiekhattar R, Shilatifard A. An operational definition of epigenetics. Genes Develop. 2009;23(7):781-3.

24. Tao R, Jin B, Guo SZ, Qing W, Feng GY, Brooks DG, Liu L, Xu J, Li T, Yan Y, He L. A novel missense mutation of the EDA gene in a Mongolian family with congenital hypodontia. J Hum Gen. 2006;51(5):498-502. 